\title{
Transient Performance, Resetting and Filtering in Nonlinear Multiple Model Adaptive Control
}

\author{
Jostein Hansen and Tor A. Johansen* \\ Department of Engineering Cybernetics, Norwegian University of Science and Technology, Trondheim, Norway.
}

\begin{abstract}
Multiple model adaptive control (MMAC) is a well established approach for implementing adaptive systems with fast transient response. This paper considers a recently developed MMAC method based on adaptive nonlinear backstepping control where the parameter estimate may be discontinuously reset based on a criterion that requires a negative jump in the associated control Lyapunov function. Particular attention is paid to transient effects due to data filtering, which must be introduced in any practical implementation of the MMAC algorithm in order to reduce the sensitivity to noise, disturbances and model uncertainty. The main contribution of this paper is insight into the robust behavior of the adaptive system resulting from the filtering, and an investigation into the trade-offs between high transient performance and robustness to uncertainty. We also suggest data filter tuning guidelines and illustrate the results using a simulation example.
\end{abstract}

\section{INTRODUCTION}

The main motivation for introducing reset of the parameter estimator in adaptive systems is to increase the transient performance without increasing the steady-state noise sensitivity. The transient performance has a strong relationship to the choice of the adaption gain matrix. The higher the values of the diagonal elements of the adaption gain matrix, the better the transient performance of the system. However, the noise sensitivity of the system increases with higher values. Introducing parameter resetting to rapidly counteract large parameter estimator errors may lead to a system having both fast transient response and a low steady-state noise sensitivity.

To cope with this problem, multiple model adaptive control (MMAC) approaches have been proposed [1], [2], [3], [4], [5], [6], and [7]. Most of the approaches are limited to linear systems, except [5], [6] and [7]. A fairly general class of systems are considered in [7], where the idea of proving stability is combined with the development of the reset algorithm. This is done by using the proposed control Lyapunov function from the adaptive control design as a criterion for performing reset. However, the analysis in [7] are based on some simplifications, in particular neglecting the effect of some filters that must be introduced in a practical implementation of the resetting algorithm in order to reduce the effect of noise, disturbances and model uncertainty. This article deals with the infirmity above.

\section{Multiple MODEL ADAPTIVE CONTROLlER WiTH}

\section{A. Adaptive controller}

\section{RESETTING}

The systems to be studied in this article are so-called parametric strict-feedback systems, [8]:

$$
\begin{gathered}
\dot{x}_{1}=x_{2}+\varphi_{1}\left(x_{1}\right)^{T} \theta \\
\vdots \\
\dot{x}_{n-1}=x_{n}+\varphi_{n-1}\left(x_{1}, x_{2}, \ldots, x_{n-1}\right)^{T} \theta \\
\dot{x}_{n}=\beta(x) u+\varphi_{n}(x)^{T} \theta \\
\text { *E-mail: }\{\text { josteih,torj\} @itk.ntnu.no }
\end{gathered}
$$

where $\theta \in \mathbb{R}^{k}$ represents an unknown parameter vector and the $\varphi_{i}$ 's represent known smooth nonlinearities. In this article the so-called tuning function design procedure is preferred, [8].

The backstepping adaptive controller and its corresponding adaptation law for the system (1) are given by

$$
\begin{aligned}
& u=\frac{\alpha_{n}(x, \hat{\theta})}{\beta(x)} \\
& \dot{\hat{\theta}}=\Gamma \tau_{n}(x, \hat{\theta})
\end{aligned}
$$

where the adaption gain matrix $\Gamma=\Gamma^{T}>0$. The control law $\alpha_{n}$ and the tuning function $\tau_{n}$ are given by the following recursive equations:

$$
\begin{aligned}
z_{i} & =x_{i}-\alpha_{i-1} \\
\alpha_{i}\left(x_{1}, \ldots, x_{i}, \hat{\theta}\right) & =-z_{i-1}-c_{i} z_{i}-w_{i}^{T} \hat{\theta} \\
& +\sum_{k=1}^{i-1} \frac{\partial \alpha_{i-1}}{\partial x_{k}} x_{k+1}+\frac{\partial \alpha_{i-1}}{\partial \hat{\theta}} \Gamma \tau_{i} \\
& +\sum_{k=2}^{i-1} \frac{\partial \alpha_{k-1}}{\partial \hat{\theta}} \Gamma w_{i} z_{k} \\
\tau_{i}\left(x_{1}, \ldots, x_{i}, \hat{\theta}\right) & =\tau_{i-1}+w_{i} z_{i} \\
w_{i}\left(x_{1}, \ldots, x_{i}, \hat{\theta}\right) & =\varphi_{i}-\sum_{k=1}^{i-1} \frac{\partial \alpha_{i-1}}{\partial x_{k}} \varphi_{k}
\end{aligned}
$$

and $z_{0}=0, \alpha_{0}=0, \tau_{0}=0, c_{i}>0$. See [8] for more details. The above adaptive control design makes the time-derivative of the following control Lyapunov function negative definite:

$$
V(x, \hat{\theta})=\frac{1}{2} z^{T} z+\frac{1}{2}(\hat{\theta}-\theta)^{T} \Gamma^{-1}(\hat{\theta}-\theta)
$$

\section{B. Ideal estimator reset criterion}

In [7] an extension of this design is introduced by allowing the parameter estimate to be reset instantaneously from $\hat{\theta}(t)$ to $\hat{\theta}\left(t^{+}\right)$, where $t^{+}$denotes an infinitely small time increment of $t$. Note that at these instants in time the parameter update law (3) does not apply, leading to a control Lyapunov function that may be discontinuous as a function of time. Suppose the parameter estimate is reset at time $t$ to a value $\hat{\theta}\left(t^{+}\right)=\hat{\theta}_{i}$. The corresponding jump in the Lyapunov function is given by

$$
\begin{aligned}
\Delta V_{i}(t) & =V\left(x(t), \hat{\theta}_{i}\right)-V(x(t), \hat{\theta}(t)) \\
& =\Delta V_{z_{i}}(t)-\frac{1}{2}\left(\hat{\theta}_{i}-\hat{\theta}(t)\right)^{T} \Gamma^{-1}\left(2 \theta-\hat{\theta}(t)-\hat{\theta}_{i}\right)
\end{aligned}
$$

where

$$
\Delta V_{z_{i}}(t)=\frac{1}{2}\left(z\left(t^{+}, \hat{\theta}_{i}\right)^{T} z\left(t^{+}, \hat{\theta}_{i}\right)-z(t)^{T} z(t)\right)
$$


A stability-preserving and performance-improving reset condition is now $\Delta V_{i}(t)<0$, as summarized in the following theorem [7]:

Theorem 1: Suppose the parameter estimate is reset at time $t$ to $\hat{\theta}\left(t^{+}\right)=\hat{\theta}_{i}$ only if $\Delta V_{i}(t)<0$. Then the adaptive control system with estimator resetting satisfies

$$
\dot{V}(t) \leq-z^{T} C z
$$

where $\dot{V}(t)$ is interpreted in the usual distributional sense when $V(t)$ is discontinuous.

Remark 1: Without any reset, the time-derivative of the Lyapunov function will be, $\dot{V}(t)=-z^{T} C z$, such that reset makes $V(t)$ decay faster.

Direct application of this theorem assumes that $\theta$ used in the expression (6) can be estimated perfectly from measurement data, and in [7] a method of estimating the unknown $\theta$ is given. However, they neglect the effect of data filters that must be incorporated in a practical algorithm in order to achieve robustness with respect to noise and uncertainty. Below, we review this method and study in detail the filter transients.

\section{Parameter estimator}

We assume a finite number of fixed parameter hypotheses $\hat{\theta}_{1}, \ldots, \hat{\theta}_{h}$ that are compared at each time instant to see which one gives the largest decrease in $\Delta V_{i}(t)$.

The first step in the development of the parameter estimator is done by filtering both sides of the parametric strict-feedback system (1)

$$
\begin{aligned}
s H_{1}(s) x_{1} & =H_{1}(s) x_{2}+H_{1}(s)\left[\varphi_{1}\left(x_{1}\right)^{T} \theta\right] \\
\vdots & \\
s H_{n-1}(s) x_{n-1} & =H_{n-1}(s) x_{n} \\
& +H_{n-1}(s)\left[\varphi_{n-1}\left(x_{1}, x_{2}, \ldots, x_{n-1}\right)^{T} \theta\right] \\
s H_{n}(s) x_{n} & =H_{n}(s)[\beta(x) u]+H_{n}(s)\left[\varphi_{n}(x)^{T} \theta\right]
\end{aligned}
$$

where $H_{i}(s)$ is typically a lowpass or bandpass filter. The main purpose of these filters is to replace differentiation operations by appropriate high-pass filters, in addition to reducing the effect of high-frequency noise, low-frequency disturbances and other model uncertainty in the estimation model. We introduce the following scalar definitions

$$
\begin{aligned}
y_{1}(t) & =s H_{1}(s) x_{1}-H_{1}(s) x_{2} \\
\vdots & \\
y_{n-1}(t) & =s H_{n-1}(s) x_{n-1}-H_{n-1}(s) x_{n} \\
y_{n}(t) & =s H_{n}(s) x_{n}-H_{n}(s)[\beta(x) u]
\end{aligned}
$$

in order to define the vector $y(t)=$ $\left[y_{1}(t), \ldots, y_{n}(t)\right]^{T}$. From (9) we have the following relationship

$$
\begin{aligned}
y(t) & =\left[\begin{array}{lll}
H_{1}(s)\left[\varphi_{1}\left(x_{1}\right)^{T} \theta\right], & \ldots, & H_{n}(s)\left[\varphi_{n}(x)^{T} \theta\right]
\end{array}\right]^{T} \\
& =H(s)\left[F(x)^{T} \theta\right]
\end{aligned}
$$

where $F^{T}(x)=\left[\varphi_{1}\left(x_{1}\right), \varphi_{2}\left(x_{1}, x_{2}\right), \ldots, \varphi_{n}(x)\right]^{T}$ and the $n \times n$-matrix $H(s)=\operatorname{diag}\left(H_{1}(s), \ldots, H_{n}(s)\right)$.

In order to study transients due to the filter initial state, which have been neglected so far, we consider a state space realization of (11). The parameter $\theta$ may be looked as if it is constant. This is possible by transforming any sudden changes of the parameter value into corresponding jumps in the filter states initial conditions.

Lemma 1: The filter (11) may be written in the regressor form:

$$
y(t)=\zeta^{T}(t) \theta+\breve{y}(t)
$$

where the $n \times 1$-vector $y(t)$ and the $n \times k$-matrix $\zeta^{T}(t)=$ $\left[\zeta_{1}(t), \zeta_{2}(t), \ldots, \zeta_{n}(t)\right]^{T}$ are given by the following two state space realizations:

$$
\begin{aligned}
\dot{x}_{y} & =A x_{y}+B F(x)^{T} \theta \\
y & =C x_{y}+D F(x)^{T} \theta \\
\dot{x}_{\zeta} & =A x_{\zeta}+B F(x)^{T} \\
\zeta^{T} & =C x_{\zeta}+D F(x)^{T}
\end{aligned}
$$

where $A, B, C$ and $D$ are given by $A=\operatorname{diag}\left(A_{1}, \ldots, A_{n}\right)$, $B=\operatorname{diag}\left(B_{1}, \ldots, B_{n}\right), C=\operatorname{diag}\left(C_{1}, \ldots, C_{n}\right)$ and $D=$ $\operatorname{diag}\left(D_{1}, \ldots, D_{n}\right)$, and $\left(A_{i}, B_{i}, C_{i}, D_{i}\right)$ are realizations of the filter transfer function $H_{i}(s)$. Further, the transient term $\breve{y}(t)$ is given as:

$$
\breve{y}(t)=C e^{A t}\left(x_{y}(0)-x_{\zeta}(0) \theta\right)
$$

Proof: Solving the two differential equations, (13) and (14) yields:

$$
\begin{aligned}
& y(t)=C\left(e^{A t} x_{y}(0)+\int_{0}^{t} e^{A(t-\tau)} B F(x(\tau))^{T} \theta d \tau\right) \\
& +D F(x(t))^{T} \theta \\
& \zeta^{T}(t)=C\left(e^{A t} x_{\zeta}(0)+\int_{0}^{t} e^{A(t-\tau)} B F(x(\tau))^{T} d \tau\right) \\
& +D F(x(t))^{T} \\
& \text { Using } \quad C \int_{0}^{t} e^{A(t-\tau)} B F(x(\tau))^{T} \theta d \tau \\
& C \int_{0}^{t} e^{A(t-\tau)} B F(x(\tau))^{T} d \tau \theta \text {, we get: } \\
& y(t)=C e^{A t} x_{y}(0)+\left(\zeta^{T}(t)-C e^{A t} x_{\zeta}(0)\right) \theta \\
& =\zeta^{T}(t) \theta+C e^{A t} x_{y}(0)-C e^{A t} x_{\zeta}(0) \theta
\end{aligned}
$$

which gives (12) and (15).

Defining the predictor

$$
\hat{y}_{i}(t)=\zeta^{T}(t) \hat{\theta}_{i}
$$

we get an expression for the prediction error:

$$
e_{i}(t)=y(t)-\hat{y}_{i}(y)=\zeta^{T}(t)\left(\theta-\hat{\theta}_{i}\right)+\breve{y}(t)
$$

Multiplying both sides of (21) by $\zeta(t)$ gives

$$
\zeta(t) e_{i}(t)=\zeta(t) \zeta^{T}(t)\left(\theta-\hat{\theta}_{i}\right)+\zeta(t) \breve{y}(t)
$$

Since the term $\zeta(t) \zeta^{T}(t)$ may be singular, equation (22) cannot in general be solved for $\theta$. One approach may be to filter both sides of (22). For simplicity, we consider a first order filter, $G(s)=1 /\left(1+\tau_{g} s\right)$ :

$$
\begin{aligned}
G(s)\left[\zeta(t) e_{i}(t)\right] & =G(s)\left[\zeta(t) \zeta^{T}(t)\right]\left(\theta-\hat{\theta}_{i}\right) \\
& +G(s)[\zeta(t) \breve{y}(t)]
\end{aligned}
$$

In order to account for transients, we consider instead state-space realizations of the filters. For convenience, first define two zero-state terms in (23) as: 


$$
\begin{aligned}
& g_{i}(t)=\frac{1}{\tau_{g}} \int_{0}^{t} e^{-\frac{t-\tau}{\tau_{g}}} \zeta(\tau) e_{i}(\tau) d \tau \\
& S(t)=\frac{1}{\tau_{g}} \int_{0}^{t} e^{-\frac{t-\tau}{\tau_{g}}} \zeta(\tau) \zeta^{T}(\tau) d \tau
\end{aligned}
$$

One way of reconstruct $\theta$, with a separate term for the transient part, is presented in the following lemma:

Lemma 2: Assuming persistence of excitation of $S(t)$, i.e. there exist an $\varepsilon>0$ such that for all $t \geq 0, S(t) \geq$ $\varepsilon I$, then the unknown parameter $\theta$ may be represented as follows:

$$
\theta=S^{-1}(t) g_{i}(t)+\hat{\theta}_{i}+\theta_{\text {trans }}(t)
$$

where transient term of (25) is

$$
\begin{aligned}
\theta_{\text {trans }}(t) & =-e^{-\frac{t}{\tau_{g}}} S^{-1}(t) S(0) \Delta \theta \\
& -S^{-1}(t) \frac{1}{\tau_{g}} \int_{0}^{t} e^{-\frac{t-\tau}{\tau_{g}}} \zeta(\tau) \breve{y}(\tau) d \tau
\end{aligned}
$$

$\Delta \theta$ is a constant vector, representing the jump in the unknown parameter $\theta$.

Proof: Using (24a) and (24b) to write (23) in time domain representation:

$$
\begin{aligned}
g_{i}(t) & =S(t)\left(\theta-\hat{\theta}_{i}\right)+e^{-\frac{t}{\tau_{g}}} S(0) \Delta \theta \\
& +\frac{1}{\tau_{g}} \int_{0}^{t} e^{-\frac{t-\tau}{\tau_{g}}} \zeta(\tau) \breve{y}(\tau) d \tau
\end{aligned}
$$

where $e^{-\frac{t}{\tau_{g}}} S(0) \Delta \theta$ is the transient term in filter $G(s)$, which accounts for jump in the unknown parameter value $\theta$. The result follows by reorganizing the terms.

Assuming $S(t) \geq \varepsilon I$, we consider the following direct estimate of $\theta$ which neglects the transient part of (25):

$$
\theta_{\text {est }}(t)=S^{-1}(t) g_{i}(t)+\hat{\theta}_{i}
$$

The overall idea of using the estimate $\theta_{\text {est }}(t)$ of the unknown parameter $\theta$ in (6) for the reset criterion, is based on the fact that $\theta_{\text {trans }}(t)$ may settle much faster than the parameter estimate $\hat{\theta}(t)$ in the update law (3) such that $\theta_{\text {est }}(t)$ will be a better estimate than $\hat{\theta}(t)$ during a transient phase. On the other hand, the accuracy of the estimate $\theta_{\text {est }}(t)$ will indeed be much more sensitive to noise than $\hat{\theta}(t)$, since the dynamics of the filters $G(s)$ and $H_{i}(s)$ are usually much faster than the dynamics of $\hat{\theta}(t)$ in (3). This may be considered to be of less importance as $\theta_{\text {est }}(t)$ is intended to be used as an estimate only during a transient phase. We remark that the estimate (28) is similar to the estimate proposed in [7], the main difference being that we consider in detail the effect of $\theta_{\text {trans }}(t)$ in the present paper.

As stated above, a form of persistence of excitation must be assumed for (24b). It can be seen from (28) that poor conditioning of $S(t)$ generally means that the estimate $\theta_{\text {est }}(t)$ is inaccurate. Moreover, the persistence excitation condition will typically hold during transients, where a reset may be profitable. On the other hand, when close to steady state, the persistence of excitation condition may not hold, but in such an instance there is no need for reset anyway. Hence, reset is not permitted when $S(t)$ is found to be too poorly conditioned.

\section{TRANSIENT ANALYSIS OF THE EFFECT OF FILTERING AND UNCERTAINTY}

\section{A. Main result}

First we define an estimate of the Lyapunov function (5) based on the estimate $\theta_{\text {est }}(t)$ from the previous section:

$$
V_{\text {est }}(x, \hat{\theta})=\frac{1}{2} z^{T} z+\frac{1}{2}\left(\hat{\theta}-\theta_{\text {est }}\right)^{T} \Gamma^{-1}\left(\hat{\theta}-\theta_{\text {est }}\right)
$$

An instantaneous jump $\hat{\theta}\left(t^{+}\right)=\hat{\theta}_{i}$ leads to an instantaneous change in $V_{\text {est }}(t)$

$$
\begin{aligned}
\Delta V_{i, e s t}(t) & =\frac{1}{2}\left(z\left(t^{+}, \hat{\theta}_{i}\right)^{T} z\left(t^{+}, \hat{\theta}_{i}\right)-z(t)^{T} z(t)\right) \\
& -\frac{1}{2}\left(\hat{\theta}_{i}-\hat{\theta}(t)\right)^{T} \Gamma^{-1}\left(2 \theta_{\text {est }}(t)-\hat{\theta}(t)-\hat{\theta}_{i}\right)
\end{aligned}
$$

The point of interest is located in the difference between the jump in the adaptive control Lyapunov function (6), and its estimated value (30) being used in the reset criterion $\Delta V_{i, e s t}(t)<0$.

It is assumed that a negative jump in the exact adaptive control Lyapunov function (5) is desirable whenever this is possible (recall Theorem 1).

Criterion 1: To ensure avoiding erroneous reset using $\Delta V_{i, e s t}(t)<0$ instead of $\Delta V_{i}(t)<0$ as the reset criterion, a negative value of $\Delta V_{i, \text { est }}(t)$ must lead to a negative value of $\Delta V_{i}(t)$. The converse is not necessary.

Using $\theta=\theta_{\text {est }}(t)+\theta_{\text {trans }}(t)$, eq. (30) can be written

$\Delta V_{i, \text { est }}(t)=\Delta V_{i}(t)+\frac{1}{2}\left(\hat{\theta}_{i}-\hat{\theta}(t)\right)^{T} \Gamma^{-1}\left(2 \theta_{\text {trans }}(t)\right)$

It is easily seen that the estimate $\Delta V_{i, e s t}(t)$ tends to the correct value $\Delta V_{i}(t)$, since $\theta_{\text {trans }}(t) \rightarrow 0$ as $t \rightarrow \infty$. The following theorem summarizes what is stated in Criterion 1 in a precise manner.

Theorem 2: Suppose

$$
\Delta V_{i, e s t}(t)<0
$$

Then no erroneous reset, according to Criterion 1, will occur if the following inequality also holds:

$$
\frac{1}{2}\left(\hat{\theta}_{i}-\hat{\theta}(t)\right)^{T} \Gamma^{-1}\left(2 \theta_{\text {trans }}(t)\right) \geq 0
$$

Notice that $\theta_{\text {trans }}(t)$ cannot be assumed to be known, so this theorem cannot be used in a direct way to formulate a better reset criterion. Instead, we pursue addition insight from this result in the following sections.

\section{B. Geometric interpretation for first order systems}

First assume for simplicity that $z$ is of dimension one, which leads to $\Delta V_{z_{i}}(t)=0$, such that (32) may be written:

$$
\begin{aligned}
& \frac{1}{2}\left(\hat{\theta}_{i}-\theta_{\text {est }}(t)\right)^{T} \Gamma^{-1}\left(\hat{\theta}_{i}-\theta_{\text {est }}(t)\right) \\
& -\frac{1}{2}\left(\hat{\theta}(t)-\theta_{\text {est }}(t)\right)^{T} \Gamma^{-1}\left(\hat{\theta}(t)-\theta_{\text {est }}(t)\right)<0
\end{aligned}
$$

which is the same as claiming $\left\|A\left(\hat{\theta}_{i}-\theta_{\text {est }}(t)\right)\right\|^{2}<$ $\left\|A\left(\hat{\theta}(t)-\theta_{\text {est }}(t)\right)\right\|^{2}$, with the Cholesky factorization $\Gamma^{-1}=A^{T} A$. For simplicity, assume equal diagonal elements in $\Gamma$, leading to the fact that the inequality can be rewritten as $\left\|\hat{\theta}_{i}-\theta_{\text {est }}(t)\right\|<\left\|\hat{\theta}(t)-\theta_{\text {est }}(t)\right\|$. This can be done without loss of generality because $\Gamma$ may be 


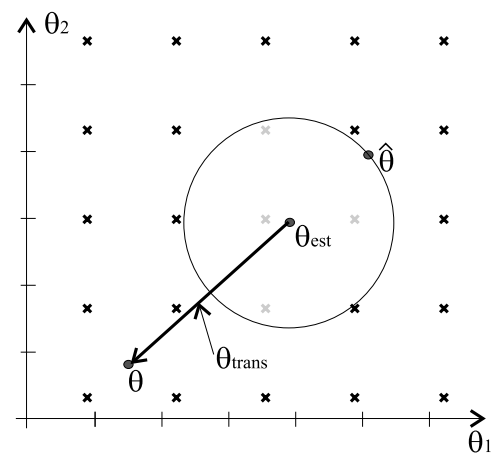

Fig. 1. Visualization of inequality (32) of Theorem 2.

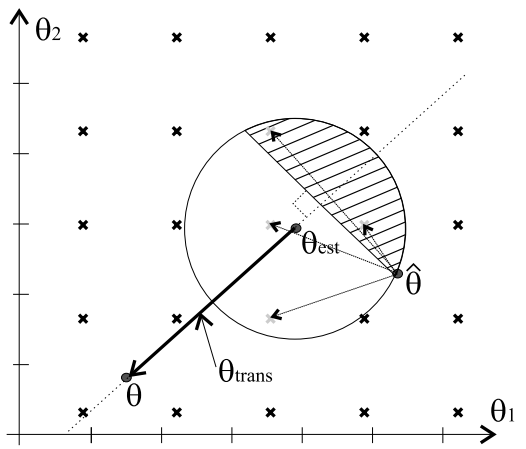

Fig. 2. Visualization of inequality (33) of Theorem 2 in terms of a forbidden region.

transformed to $c I$ by linear transformation of the coordinate axis in the $\theta$-system. The given inequality, and indeed (32), is satisfied if the estimator reset candidate $\hat{\theta}_{i}$ is located inside the circle centered in $\theta_{\text {est }}(t)$ with radius $\left\|\hat{\theta}(t)-\theta_{\text {est }}(t)\right\|$. This is depicted in Figure 1, where the estimator reset candidates satisfying (32) are made gray, in contrast to the black ones not satisfying (32).

Further, for Theorem 2 to hold, the inequality (33) must hold as well. This inequality may be seen as the "forbidden" zone inside the circle, and is visualized as the shaded area in Figure 2.

According to (33), the angle between the two vectors $\theta_{\text {trans }}(t)$ and $\hat{\theta}_{i}-\hat{\theta}(t)$ must be between $\pm 90^{\circ}$, for any reset candidate $\hat{\theta}_{i}$ if an erroneous reset is to be avoided.

\section{Geometric interpretation for systems of higher order}

Next assume that $\Gamma$ may not have equal diagonal elements, and $z$ may not be scalar. The latter means that $\Delta V_{z_{i}}(t)$ may be different from zero. The inequality in (32) may be written as

$$
\begin{aligned}
& \left\|A\left(\hat{\theta}_{i}-\theta_{\text {est }}(t)\right)\right\|^{2}+\left\|z\left(t^{+}\right)\right\|^{2}-\|z(t)\|^{2} \\
& <\left\|A\left(\hat{\theta}(t)-\theta_{\text {est }}(t)\right)\right\|^{2}
\end{aligned}
$$

and (33) becomes

$$
\frac{1}{2}\left(A\left(\hat{\theta}_{i}-\hat{\theta}(t)\right)\right)^{T}\left(2 A \theta_{\text {trans }}(t)\right) \geq 0
$$

This leads to two different situations, one for where $\Delta V_{z_{i}}(t)$ is negative and another for where it is positive. Consider first the positive case

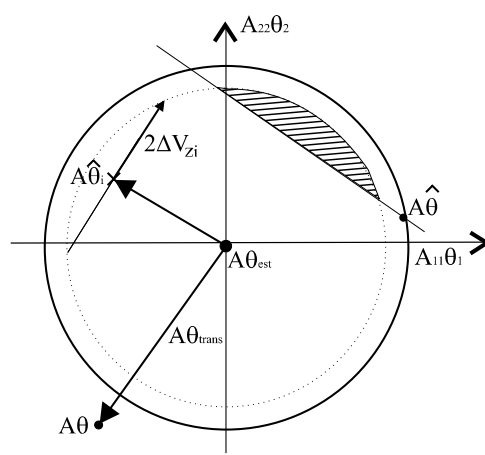

Fig. 3. Visualization of inequality (33) of Theorem 2 with positive jump $\Delta V_{z_{i}}(t)>0$, as a forbidden region.

$$
\left\|A\left(\hat{\theta}_{i}-\theta_{\text {est }}(t)\right)\right\|^{2}+2 \Delta V_{z_{i}}(t)<\left\|A\left(\hat{\theta}(t)-\theta_{\text {est }}(t)\right)\right\|^{2}
$$

Hence, a similar geometric interpretation can be given in a space that is similar to the $\theta$-space in terms of the transformation matrix $A$, see Figure 3 for the case when $\Delta V_{z_{i}}(t)>0$.

The other case where $\Delta V_{z_{i}}(t)<0$ is interpreted in a similar way, but by expanding the circular region, hence left out to save space. If $\theta$ has dimension higher than 2 , the geometric interpretations may be generalized in a straightforward manner using hyper-spheres and hyperplanes.

\section{Resetting criterion revisited}

In the context of the above geometric interpretation, avoiding erroneous resets due to filter transients essentially amounts to avoiding reset candidates within the "forbidden region" that may violate (33) in Theorem 2, shown as the hatched half-moon shaped area in Figure 3. We first remark that exact knowledge of this area requires knowledge of the direction of the vector $\theta_{\text {trans }}(t)$, which links the points $\theta$ and $\theta_{\text {est }}(t)$. This information is not generally available.

However, from the discussion in section III-B, it is clear that if the point $\theta_{\text {est }}(t)$ is located on a straight line between $\theta$ and $\hat{\theta}(t)$ and initially $\hat{\theta}(0)=\theta_{\text {est }}(0)$, the "forbidden region" degenerates to a point which means that no erroneous reset will ever occur. $\hat{\theta}(0)=\theta_{\text {est }}(0)$ essentially mean that $\hat{\theta}(t)$ has reached its steady state value $\theta$ before a new sudden change of $\theta$ takes place, where our time measurement is reset to $t=0$. Note that the necessity of letting $\hat{\theta}(t)$ reach its steady state, may be fulfilled without having to wait for its slow dynamics. This is so because resets may have taken place during its settlement, hence speeding up its dynamic behavior.

At a first glance, it may seem unlikely that $\theta_{\text {est }}(t)$ will be confined to such a straight line, but it turns out that this may in fact be a close to typical situation, as pointed out in the following theorem.

Theorem 3: Assume the dynamics of the closed-loop system (1)-(3) without reset is viewed to be infinitely slow compared to the system's data filter dynamics. Further assume the filters, $H_{1}(s), H_{2}(s), \ldots, H_{n}(s)$ are of order one and equal. If $S(t) \geq \varepsilon I$ where $\varepsilon>0$ for all $t \geq 0$, then the trajectory of $\theta_{\text {est }}(t)$ is a straight line between $\theta_{\text {est }}(0)$ and $\theta$.

Proof: Since $\theta=\theta_{\text {trans }}(t)+\theta_{\text {est }}(t)$ is constant, we have to prove that $\theta_{\text {trans }}(t)$ evolves in straight lines. Since 
the closed-loop dynamics of the system is assumed to be infinitely slow, we may view $S(t)=S(0)=\dot{S}$ and $\zeta(t)=\dot{\zeta}$ as constants. Further, due to the filter assumptions in the theorem, $C=I, A=-\frac{1}{\tau_{h}} I$ and $B=\frac{1}{\tau_{h}} I$, where $\tau_{h}$ is the time constant of filters $H_{i}(s)$, we may rewrite (26) as:

$$
\begin{aligned}
\theta_{\text {trans }}(t) & =-e^{-\frac{t}{\tau_{g}}} \Delta \theta \\
& -\frac{1}{\tau_{g} \tau_{h}} \int_{0}^{t} e^{-\frac{t-\tau}{\tau_{g}}} S^{-1} \xi e^{-\frac{\tau}{\tau_{h}} I} \dot{F}^{T} \Delta \theta d \tau
\end{aligned}
$$

Note that the definition in (15) and the assumption of infinite slow dynamics, hence $F^{T}(x)=F^{T}$, has also been used. (38) may be seen as a first order low pass filtering of straight line behavior input signal, whose transient terms due to initial conditions are moving in parallel with this input. This leads to an output result $\theta_{\text {trans }}(t)$ also with straight line behavior, hence $\theta_{\text {est }}(t)$ moves in straight lines.

It follows that the straight line assumption is not severely unrealistic such that the "forbidden region" is typically a small subset of the circle in the geometric interpretation in Figure 2. This will be further discussed and justified in the context of the example in section IV.

\section{CASE STUdY}

\section{A. Augmented quarter car model}

A case study that illustrates the concepts given in this article is an extension of the wheel slip control system presented in [7]. The simple model in [7] is a quarter car model. The state of interest is the wheel slip $\lambda$, defined as:

$$
\lambda=\frac{v-\omega r}{v}
$$

which is the normalized difference between horizontal speed $v$ and the speed of the wheel perimeter $\omega r$. Due to the shape of the friction curve, a slip value of 0.15 is chosen as the setpoint value which gives close to maximum friction, see [9] for more details.

In addition to the simple quarter car model in [7], the dynamics of the actuator is also included. The system may be presented as follows

$$
\begin{aligned}
\dot{x}_{1} & =k \check{x}_{2}+\varphi_{1,1}\left(x_{1}\right) \theta_{1} \\
\tau_{a} \dot{\check{x}}_{2} & =u-\check{x}_{2}
\end{aligned}
$$

where $x_{1}$ represents the wheel slip error, $x_{1}=\lambda-0.15$, $k \check{x}_{2}$ represents the brake force produced by the actuator, and $u$ is the clamping force commanded to the actuator. The parameter $k$ is an unknown factor that represents the conversion of clamping force from the actuator output to the actual brake force in the quarter car system. $k$ can then be seen as a gain that connects the two sub-systems (40) and (41), whose domain is assumed to be $k \in[0.8,1.2]$. A realistic choice of the actuator dynamics time constant $\tau_{a}$ is $0.08 \mathrm{sec}$. The nonlinear function $\varphi_{1,1}\left(x_{1}\right)$ represents the friction curve as in [7]:

$$
\begin{aligned}
\varphi_{1,1}\left(x_{1}\right) & =-\left(1+0.05\left(0.85-x_{1}\right)\right) \\
& \left(1.02-e^{\left(-20 x_{1}-3\right)}-0.2 x_{1}\right)
\end{aligned}
$$

The domain of the road/tyre friction coefficient $\theta_{1}$, is from $\theta_{1}=0$ indicating no tyre/road surface friction to $\theta_{1}=$ 1 corresponding to maximum friction (dry asphalt surface).

The model (40) - (41) is not in standard parametric strictfeedback form (1), and has to be transformed to this form to be controlled by the strategy described in Section II. By defining $x_{2}=k \check{x}_{2}$, and assuming $\frac{\tau_{a}}{k} \approx \tau_{a}$ due to the relatively little uncertainty in $k \approx 1,(40)$ and (41) may be written as

$$
\begin{aligned}
& \dot{x}_{1}=x_{2}+\varphi_{1,1}\left(x_{1}\right) \theta_{1} \\
& \dot{x}_{2}=\frac{1}{\tau_{a}} u-\frac{1}{k} \frac{1}{\tau_{a}} x_{2}
\end{aligned}
$$

which in turn can be written in parametric strict-feedback form (1) using $\beta(x)=\frac{1}{\tau_{a}}, \varphi_{1}^{T}\left(x_{1}\right)=\left[\varphi_{1,1}\left(x_{1}\right), 0\right]$, $\varphi_{2}^{T}\left(x_{2}\right)=\left[0, \varphi_{2,2}\left(x_{2}\right)\right]$ and $\theta^{T}=\left[\theta_{1}, \theta_{2}\right]$, where $\theta_{2}=\frac{1}{k}$ and $\varphi_{2,2}\left(x_{2}\right)=-\frac{1}{\tau_{a}} x_{2}$.

\section{B. Simulation scenario}

The simulation is based on a situation of a car braking on a heterogeneous road surface, for example asphalt that is partly covered with ice or water. This leads to rapidly changing road/tyre friction coefficient $\theta_{1}$. In addition to this, the amount of force transferred from the actuators to the brake pads is given by the force gain coefficient $\theta_{2}$. The force transmission may change almost instantaneously due to leakage in the hydraulics or the brake pads becoming wet. A worst case scenario is when both $\theta_{1}$ and $\theta_{2}$ change their values at the same time. The scenario to be simulated is represented by the step changes in $\theta$ as shown by the solid line in Figure 5

\section{Reset algorithm and tuning}

The adaptive controller may be tuned by first disconnecting the reset algorithm. The parameters of the adaptive controller are chosen as $c_{1}=1, c_{2}=30$ and $\Gamma=$ $0.5 I$, leading to satisfactory adaptive system performance. The filters $G(s), H_{1}(s), \ldots, H_{n}(s)$ of the estimator, are all chosen to be low-pass filters of order one, all with time constants equal to $85 \mathrm{~ms}$. This is slow enough to reduce noise sensitivity of the estimate $\theta_{\text {est }}(t)$, while fast enough such that the transient performance is not slowed down significantly.

In this example $\theta \in \mathbb{R}^{2}$, which means that the choice of parameter reset candidates is made as a grid pattern in the plane. The "density" of the pattern must be selected to address the tradeoff between noise sensitivity and transient performance. As the density of the grid pattern increases the parameter estimate approaches the behavior of using $\theta_{\text {est }}(t)$ directly as an estimator in the adaptive system. As the grid pattern becomes more coarse, only large transients will benefit from the resetting strategy. The grid pattern used in this example is in Figure 6 indicated with small crosses in a $3 \times 5$ pattern.

\section{Simulation results}

Figure 4 shows the value of the Lyapunov function as a function of time for a simulation of the adaptive system with and without reset. Even though no safeguard regarding avoidance of erroneous reset is used, the resulting Lyapunov function response in Figure 4 shows no signs of that such an occurrence has taken place.

By comparing the two situations for system with and without reset in Figure 5, the fast filter dynamics greatly improve the transient performance without increasing the noise sensitivity, see also Figure 6. The effect of filtering leads to the parameter estimate making several small jumps in the right direction rather than one big jump directly to the right estimate (or possibly the wrong estimate due to noise and other uncertainty).

It can be verified that $S(t)$ and $\zeta(t)$ does not vary much during the transients. In the example, $\theta_{\text {est }}(t)$ does indeed 


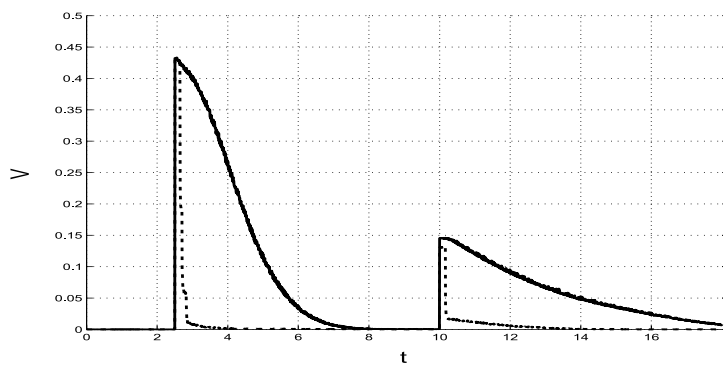

Fig. 4. Lyapunov function with (dotted) and without (solid) reset.
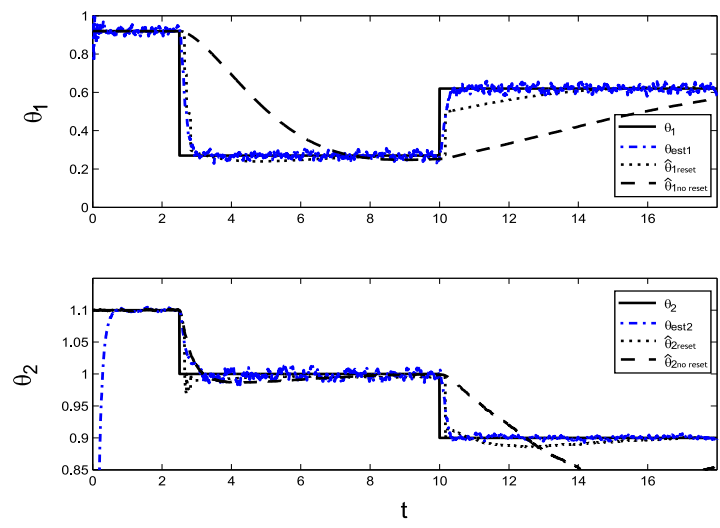

Fig. 5. Parameter adaption with and without reset.

seem to move roughly on a straight line, cf. Figure 6, due to tuning of the data filters being made in accordance with the guidelines derived above. Since the grid pattern is fairly coarse, $\hat{\theta}(t)$ is somewhat off the line between $\theta$ and $\theta_{\text {est }}(t)$ most of the time. Still, no erroneous resets were made in the simulation example, which is the typical situation with the present tuning. Immediately after a reset has taken place, there may be a short time interval when the "forbidden region" is quite large. However, the likelihood of an erroneous reset during this phase is still fairly small as the circle containing all reset candidates becomes smaller, typically containing only the one to which a reset has already been made.

\section{Conclusions}

Introducing parameter resetting in nonlinear adaptive control may greatly improve the transient performance without increasing the systems noise sensitivity significantly. This article has studied the effect of data filters in MMAC. Such filters introduce transients that complicates the estimation of jumps in the control Lyapunov function that results from parameter estimate resets. We provide insight into causes for erroneous resets due to such filter transients, addressing the inherent tradeoff between rapid transient response, and model and data uncertainty. Data filter tuning guidelines have been proposed and shown to work well in simulations.

\section{ACKNOWLEDGEMENTS}

This work was in part sponsored by the European Commission through the Research Training Network MAC

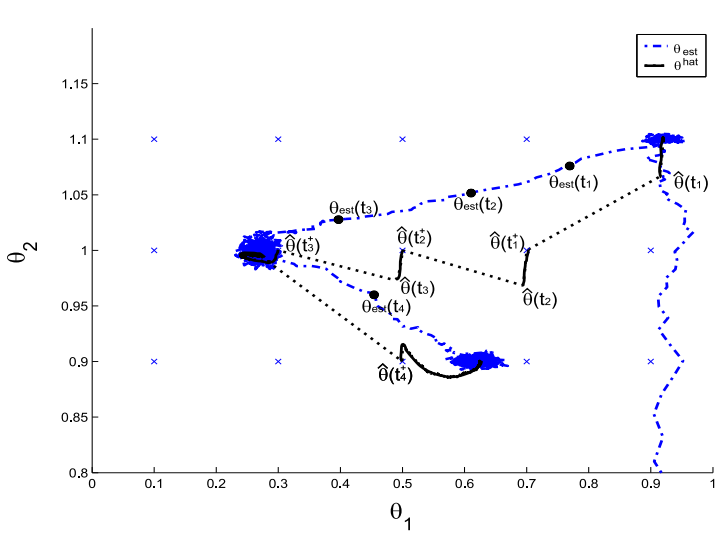

Fig. 6. Parameter adaption evolving in the two dimensional $\theta$-space. Reset is performed at $t_{1}=2.2 \mathrm{~s}, t_{2}=2.3 \mathrm{~s}, t_{3}=2.4 \mathrm{~s}$ and $t_{4}=10.1 \mathrm{~s}$.
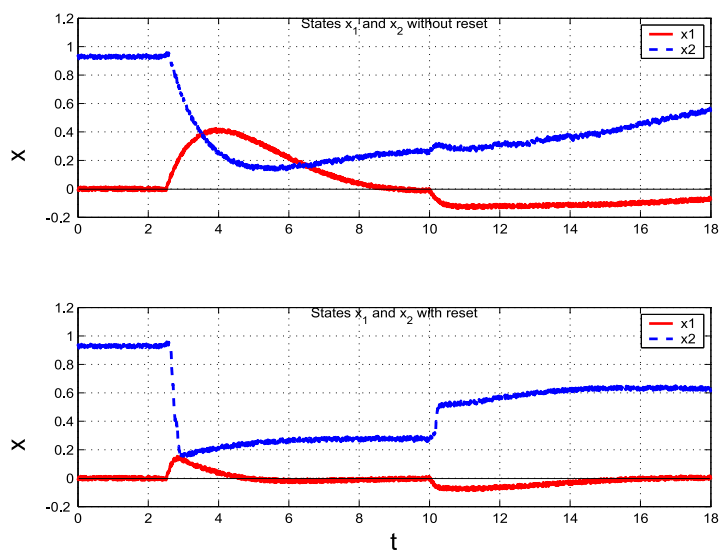

Fig. 7. State trajectories, with and without reset. Note that $x_{1}=0$ is the reference for $x_{1}$.

(Multi Agent Control)

\section{REFERENCES}

[1] R. H. Middleton, G. C. Goodwin, D. J. Hill, and D. Q. Mayne, "Design issues in adaptive control," IEEE Trans. Automatic Control, vol. 33, pp. 50-58, 1988.

[2] P. V. Zhivoglyadov, R. H. Middleton, and M. Fu, "Localization based switching adaptive control for time-varying discrete-time systems," IEEE Trans. Automatic Control, vol. 45, pp. 752-756, 2000.

[3] K. S. Narendra and J. Balakrishnan, "Improving transient response of adaptive control systems using multiple models and switching," IEEE Trans. Automatic Control, vol. 39, pp. 1861-1866, 1994.

[4] K. S. Narendra and J. Balakrishnan, "Adaptive control using multiple models," IEEE Trans. Automatic Control, vol. 42, pp. 171-187, 1997.

[5] K. S. Narendra and K. George, "Adaptive control of simple nonlinear systems using multiple models," Proc. Amer. Contr. Conf., pp. 1779$1784,2002$.

[6] J. Kalkkuhl, T. A. Johansen, J. Lüdemann, and A. Queda, "Nonlinear adaptive backstepping with estimator resetting using multiple observers," in Proc. Workshop on Hybrid Systems, Computation and Control, Rome, 2001.

[7] J. Kalkkuhl, T. A. Johansen, and J. Lüdemann, "Improved transient performance of nonlinear adaptive backstepping using estimator resetting based on multiple models," IEEE Transactions on Automatic Control, vol. 47, pp. $136-140$, Jan 2002.

[8] M. Krstic, I. Kanellakopoulos, and P. Kokotovic, Nonlinear and Adaptive Control Design, John Wiley and sons, Inc, 1995.

[9] J. Lüdemann, Heterogeneous and Hybrid Control with Application in Automotive Systems, Ph.D. thesis, University of Glasgow, 2002. 